\title{
Terpene Esters from Natural Products: Synthesis and Evaluation of Cytotoxic Activity
}

\author{
MAURICIO M. VICTOR ${ }^{1,2}$, JORGE M. DAVID ${ }^{1,2}$, MARIA C.K. SAKUKUMA ${ }^{1,2}$, LETÍCIA \\ V. COSTA-LOTUFO ${ }^{3,4}$, ANDREA F. MOURA ${ }^{3}$ and ANA J. ARAÚJO ${ }^{3,5}$ \\ ${ }^{1}$ Instituto de Química, Universidade Federal da Bahia, Depto de Química Orgânica, Rua Barão \\ do Jeremoabo, s/n, Campus de Ondina, Ondina, 40170-115 Salvador, BA, Brazil \\ ${ }^{2}$ Instituto Nacional de Ciência e Tecnologia/INCT de Energia e Ambiente,Universidade Federal \\ da Bahia/UFBA, Rua Barão de Geremoabo, 147, Campus de Ondina, 40170-290 Salvador, BA, Brazil \\ ${ }^{3}$ Departamento de Fisiologia e Farmacologia, Universidade Federal do Ceará, Centro de Ciências da \\ Saúde, Av. Coronel Nunes de Melo, 1127, Rodolfo Teófilo, 60430-270 Fortaleza, CE, Brazil \\ ${ }^{4}$ Departamento de Farmacologia, Universidade de São Paulo, Av. Professor Lineu Prestes, \\ 1524, Cidade Universitária, Butantã, 05508-900 São Paulo, SP, Brazil \\ ${ }^{5}$ Universidade Federal do Piaú, Av. São Sebastião, 2819, São Benedito, Campus \\ Ministro Reis Velloso, 64202-020 Parnaíba, PB, Brazil
}

Manuscript received on November 16, 2016; accepted for publication on February 22, 2017

\begin{abstract}
Natural steroids and triterpenes such as $\beta$-sitosterol, stigmasterol, lupeol, ursolic and betulinic acids were transformed into its hexanoic and oleic esters, to evaluate the influence of chemical modification towards the cytotoxic activities against tumor cells. The derivatives were evaluated against five tumor cell lines [OVCAR-8 (ovarian carcinoma); SF-295 (glioblastoma); HCT-116 (colon adenocarcinoma); HL-60 (leukemia); and PC-3 (prostate carcinoma)] and the results showed only betulinic acid hexyl ester exhibits cytotoxic potential activity.
\end{abstract}

Key words: antitumor activity, chemical modification, derivative synthesis, natural products esters.

\section{INTRODUCTION}

Cancer is a generic term used for a large group of diseases that can affect any part of the body, which is also described as malignant tumors and neoplasms. According World Health Organization, it accounted to 8.2 million deaths worldwide in 2012 (13\% of all deaths) with 14.1 million new cases (WHO 2016). Main risk in cancer treatment is multidrug resistance, when cell lose their sensitivity to chemotherapeutics. Due this, actions

Correspondence to: Mauricio Moraes Victor

E-mail: mmvictor@ufba.br against cancer are focused on developing and reinforcing cancer control programs and also to the search alternative treatments.

The therapeutic use of active natural compounds or its derivatives as anticancer agents represents nowadays a highly-investigated research field. Triterpenic acids exhibit unique and important biological and pharmacological activities, including anti-inflammatory, antimicrobial, antiviral, cytotoxic, and cardiovascular effects (Silva et al. 2012). The total synthesis of this class of natural products is still not an easy strategy to obtain 
multigram quantities for in vivo biological screening and evaluated assays. For example, commercial betulinic acid is prepared by chemical selective oxidation of the alcohol derivative (betulin) and it is also isolated in good yields together the isomers ursolic and oleanolic acids from a restricted group of plants. Eriope blanchetii, a shrub belonging to the Lamiaceae family, is one of them (David et al. 2001).

Important classes of natural compounds or its derivatives are esters (Azimova 2013). This class of compounds shows variety of structural types leading to a wide spectrum of biological activity, including aliphatic and aromatic moieties. Although its biological role in plants has not been fully studied, a plethora of biological activities have been appointed, such as antibacterial, antifungal, estrogenic, antiestrogenic, inhibition of testosterone secretion, immunological, anti-inflammatory, cytotoxic, and ionophoretic properties, relaxant effect, vasodilatory effect, antagonist of calcium, etc. Derivative esters of natural products can be obtained from natural sources (Carvalho et al. 2001), but its use as lead compounds for medicinal chemistry gives an idea of the potential of this approach. As selected example of lead compound derivated from betulinic acid spawned the drug Bevirimat $^{\circledR}$ (2 and 1, respectively, Fig. 1), which has been showed to be specific inhibitors of HIV-1 entry (Qian et al. 2010). According this strategy, this article described our work in syntheses of specific acyl derivatives of some natural products, and evaluation of their antiproliferative activities against some tumor cell lines.

\section{EXPERIMENTAL}

\section{GENERAL EXPERIMENTAL PROCEDURES}

DIC, DMAP, MTT, and stigmasterol were purchased from Sigma-Aldrich and used without further purification. $\beta$-Sitosterol was used as a commercial mixture with stigmasterol (70:30, respectively, MP Biomedicals). Betulinic and ursolic acids were previously isolated from Eriope blanchetti (Lamiaceae). Lupeol was obtained from the hexane extract of the roots of Bowdichia virgilioides (Fabaceae) by silica gel conventional column chromatography. Dichlorometane was refluxed with $\mathrm{CaH}_{2}$ and distilled prior to use. All reactions were performed under argon atmosphere. Analytical thin layer chromatography (TLC) was performed on E. Merck TLC plates pre-coated with silica gel 60 F254 (250 $\mu \mathrm{m}$ thickness). Visualization was accomplished using UV light and potassium permanganate solution. Column chromatography was performed on silica gel 60-230 mesh. The melting points were uncorrected and determined on a MQAPF-302 apparatus. IR spectra were measured using a Shimadzu IR-Affinity 1 spectrophotometer. Nuclear magnetic resonance spectra were recorded on Varian (Inova-500) $500 \mathrm{MHz}$ spectrometer in deuterated solvents.

\section{ISOLATION OF LUPEOL 4 FROM ROOTS OF}

BOWDICHIA VIRGILIOIDES (FABACEAE)

Dried roots of B. virgilioides (480 g) was powdered and submitted to maceration with $2 \mathrm{~L}$ of $\mathrm{MeOH}$. The $\mathrm{MeOH}$ extract $(40.7 \mathrm{~g})$ was partitioned between hexane: $\mathrm{MeOH} / \mathrm{H}_{2} \mathrm{O}(5 \%)$ and the soluble fraction of hexane obtained $(5.02 \mathrm{~g})$ was submitted to a silica gel $60 \mathrm{CC}$ employing mixtures of hexane:EtOAc. The fractions eluted with $90 \%$ hexane $(430 \mathrm{mg})$ was reactive in Libermann-Buchard reagent and purified in a Sephadex LH-20 column eluted with DCM:MeOH (1:1). This procedure permitted to obtain $153 \mathrm{mg}$ of lupeol 4. This triterpene was identified by mp, IR and NMR spectra, comparing with the literature data (Mahato and Kundu 1994).

\section{GENERAL PROCEDURE FOR THE SYNTHESIS}

OF COMPOUNDS 2A,B-6A,B (REACTIONS WERE PERFORMED IN A 2 ML VIAL)

To a solution of appropriate substrate $(0.02$ mmol) in dichloromethane was added a solution 

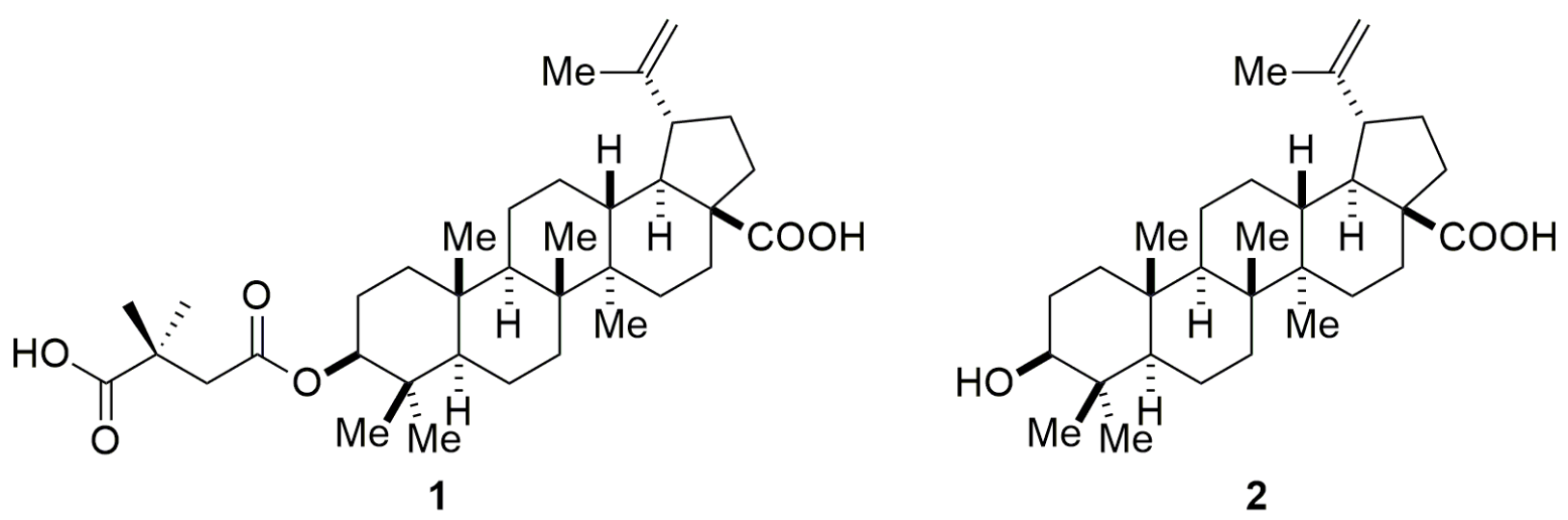

Figure 1 - Betulinic acid and its derivative Bevirimat ${ }^{\circledR}$ (2 and 1, respectively).

of DMAP (4.9 mg, $0.04 \mathrm{mmol})$ and DIC (5.1 $\mathrm{mg}, 0.04 \mathrm{mmol})$ in $0.5 \mathrm{~mL}$ of dichloromethane via cannula. Thereafter a solution of appropriate acid $(0.04 \mathrm{mmol})$ in dichloromethane $(0.5 \mathrm{~mL})$ at room temperature was added. After stirring for 24-48 hours at the same temperature, the reaction was stopped by diluting with dichloromethane, followed by filtration of solids and concentrated in a vacuum. Purification by column chromatography in silica-gel eluted with ethyl acetate:hexane furnished the desired product.

\section{Betulinyl hexanoate (2a)}

Mass obtained: $10.0 \mathrm{mg}, 94 \%$ yield; rf 0.66 in EtOAc:hexane (20:80); $[\alpha]_{\mathrm{D}}^{25}+3.0\left(c 1.0, \mathrm{CHCl}_{3}\right)$; FTIR $(\mathrm{KBr}) v / \mathrm{cm}^{-1} 3300,2856,1728,1458,1296$;

${ }^{1} \mathrm{H}$ NMR (500 MHz, $\left.\mathrm{CDCl}_{3}\right) \delta 5.30(\mathrm{~s}, 1 \mathrm{H}), 4.74$ (s, 1H), 4.62 (s, 1H), 4.48 (dd, 1H, J 10.9, $5.5 \mathrm{~Hz}$, 1H), $3.03-2.96(\mathrm{~m}, 1 \mathrm{H}), 2.40-2.18(\mathrm{~m}, 5 \mathrm{H}), 2.02$ $-1.94(\mathrm{~m}, 2 \mathrm{H}), 1.70(\mathrm{~s}, 3 \mathrm{H}), 1.67-1.58(\mathrm{~m}, 6 \mathrm{H})$, $1.56-1.48(\mathrm{~m}, 2 \mathrm{H}), 1.48-1.26(\mathrm{~m}, 14 \mathrm{H}), 1.23-1.19$ (m, 2H), 0.98 (s, 3H), 0.95 (s, 3H), 0.90 (t, 3H, J $11.0 \mathrm{~Hz}), 0.87-0.82(\mathrm{~m}, 9 \mathrm{H}) ;{ }^{13} \mathrm{C} \mathrm{NMR}(125 \mathrm{MHz}$, $\left.\mathrm{CDCl}_{3}\right) \delta 181.87,173.48,149.96,109.87,80.31$, 58.41, 56.37, 55.56, 50.56, 49.35, 38.44, 38.41, $37.86,37.24,37.10,34.78,34.32,32.16,31.20$, $31.06,30.54,29.69,27.96,25.49,24.79,24.31$,
$23.74,22.28,20.89,18.25,16.52,16.03,14.66$, $13.86,13.82,13.80$.

Betulinyl oleate (2b)

Mass obtained: $11.5 \mathrm{mg}, 80 \%$ yield; rf 0.55 in EtOAc:hexane (20:80); $[\alpha]_{\mathrm{D}}{ }^{25}+14.0\left(\mathrm{c} 1.0, \mathrm{CHCl}_{3}\right)$; FTIR (KBr) $v / \mathrm{cm}^{-1}$ 2999, 1732, 1643 1452; ${ }^{1} \mathrm{H}$ NMR (500 MHz, $\left.\mathrm{CDCl}_{3}\right) \delta 5.38-5.31(\mathrm{~m}, 2 \mathrm{H})$, 4.75 (s, 1H), 4.62 (s, 1H), 4.48 (dd, 1H, J 10.8, 5.5 $\mathrm{Hz}), 3.07-2.93(\mathrm{~m}, 1 \mathrm{H}), 2.33-2.26(\mathrm{~m}, 2 \mathrm{H}), 2.08$ $-1.91(\mathrm{~m}, 4 \mathrm{H}), 1.70(\mathrm{~s}, 3 \mathrm{H}), 1.68-1.54(\mathrm{~m}, 4 \mathrm{H})$, $1.53-1.37(\mathrm{~m}, 6 \mathrm{H}), 1.36-1.22(\mathrm{~m}, 36 \mathrm{H}), 0.98$ (s, 3H), 0.95 (s, 3H), 0.89 (t, 3H, J $6.9 \mathrm{~Hz}), 0.86$ (s, 3H), 0.84 (s, 3H), 0.83 (s, 3H); ${ }^{13} \mathrm{C}$ NMR (125 $\left.\mathrm{MHz}, \mathrm{CDCl}_{3}\right) \delta 173.62,150.32,129.97,129.73$, 109.72, 80.59, 77.22, 76.97, 56.32, 55.46, 50.45, $49.31,46.92,42.45,40.74,38.41,37.85,37.15$, $37.02,34.83,34.29,32.16,31.89,30.57,29.75$, $29.70,29.67,29.50,29.31,29.29,29.14,29.09$, $27.97,27.21,27.15,25.49,25.14,23.74,22.66$, $20.88,19.34,18.18,16.53,16.15,16.03,14.67$, 14.06 .

\section{Ursolyl hexanoate (3a)}

Mass obtained: $10.0 \mathrm{mg}, 90 \%$ yield; rf 0.70 in EtOAc:hexane (20:80); $[\alpha]_{\mathrm{D}}^{25}+8.0\left(c 1.0, \mathrm{CHCl}_{3}\right)$; FTIR (KBr) $v / \mathrm{cm}^{-1} 3414,2856,1730,1485,1240$; ${ }^{1} \mathrm{H}$ NMR (500 MHz, $\left.\mathrm{CDCl}_{3}\right) \delta 5.29-5.25(\mathrm{~m}, 1 \mathrm{H})$, 
4.54 (dd, 1H, J 10.4, $5.8 \mathrm{~Hz}), 2.32$ (t, 2H, J 7.3 Hz), $2.22(\mathrm{~d}, 1 \mathrm{H}, J 11.5 \mathrm{~Hz}), 2.07$ (s, 1H), $2.03(\mathrm{dd}, 1 \mathrm{H}$, $J$ 13.4, $4.3 \mathrm{~Hz}), 1.78-1.59$ (m, 9H), $1.58-1.48$ $(\mathrm{m}, 4 \mathrm{H}), 1.46-1.22(\mathrm{~m}, 16 \mathrm{H}), 1.14(\mathrm{~s}, 3 \mathrm{H}), 1.11$ (s, 3H), $1.01-0.98(\mathrm{~m}, 3 \mathrm{H}), 0.93(\mathrm{t}, 3 \mathrm{H}, J 6.1 \mathrm{~Hz})$, $0.91-0.85(\mathrm{~m}, 6 \mathrm{H}), 0.82(\mathrm{~s}, 3 \mathrm{H}) ;{ }^{13} \mathrm{C}$ NMR $(125$ $\left.\mathrm{MHz}, \mathrm{CDCl}_{3}\right) \delta 182.33,173.56,137.97,125.78$, 80.59, 59.56, 55.36, 52.66, 47.96, 47.50, 41.99, $39.56,39.05,38.85,38.32,38.16,37.74,36.94$, 36.72 , 34.78, 32.91, 31.33, 31.21, 30.61, 29.65, $28.09,28.02,24.80,24.13,23.60,23.56,23.30$, $22.26,21.10,18.19,17.11,16.97,16.74,15.49$, 13.82 .

\section{Ursolyl oleate (3b)}

Mass obtained: $7.0 \mathrm{mg}, 90 \%$ yield; rf 0.65 in EtOAc:hexane (20:80); $[\alpha]_{\mathrm{D}}{ }^{25}+19.0\left(c 1.0, \mathrm{CHCl}_{3}\right)$; FTIR (KBr) $v / \mathrm{cm}^{-1} 3338,2926,1710,1619 ;{ }^{1} \mathrm{H}$ NMR $\left(500 \mathrm{MHz}, \mathrm{CDCl}_{3}\right) \delta 5.36-5.32(\mathrm{~m}, 2 \mathrm{H})$, $5.26-5.23(\mathrm{~s}, 1 \mathrm{H}), 4.50(\mathrm{dd}, 1 \mathrm{H}, J 10.5,5.7 \mathrm{~Hz})$, 2.28 (td, $1 \mathrm{H}, J 7.5,3.3 \mathrm{~Hz}), 2.19$ (d, $1 \mathrm{H}, J 11.5 \mathrm{~Hz})$, $2.04-1.98(\mathrm{~m}, 4 \mathrm{H}), 1.94-1.82(\mathrm{~m}, 3 \mathrm{H}), 1.76-$ $1.58(\mathrm{~m}, 6 \mathrm{H}), 1.55-1.45(\mathrm{~m}, 4 \mathrm{H}), 1.43(\mathrm{~s}, 3 \mathrm{H})$, $1.38-1.20(\mathrm{~m}, 32 \mathrm{H}), 1.14(\mathrm{~s}, 6 \mathrm{H}), 1.12(\mathrm{~s}, 3 \mathrm{H})$, 0.97 (s, 3H), 0.93 (t, 3H, J 6.1 Hz), $0.91-0.86$ (m, $3 \mathrm{H}), 0.82(\mathrm{~s}, 3 \mathrm{H}) ;{ }^{13} \mathrm{C}$ NMR $\left(125 \mathrm{MHz}, \mathrm{CDCl}_{3}\right)$ $\delta 173.59,137.96,129.97,129.73,125.78,80.58$, 59.54, 55.33, 52.67, 47.94, 47.49, 41.99, 39.53, $39.05,38.84,38.30,38.15,37.73,36.91,36.71$, 34.82 , 34.38, 32.91, 31.89, 31.22, 30.61, 29.75, $29.67,29.50,29.31,29.29,29.14,29.09,28.10$, $28.01,27.21,27.15,25.14,24.97,24.15,23.60$, $23.54,23.30,22.65,21.13,18.19,17.09,16.99$, $16.77,15.51,14.23,14.06$.

\section{Lupenyl hexanoate (4a)}

Mass obtained: $20.0 \mathrm{mg}, 72 \%$ yield; rf 0.50 in EtOAc:hexane (20:80), $[\alpha]_{\mathrm{D}}^{25}+31.0\left(c 1.0, \mathrm{CHCl}_{3}\right)$, lit. (Brum et al. 1998) $[\alpha]_{\mathrm{D}}^{25}+31.0\left(c 1.0, \mathrm{CHCl}_{3}\right)$; $(\mathrm{KBr}) v / \mathrm{cm}^{-1} 2926,1730,1691,1541 ;{ }^{1} \mathrm{H} \mathrm{NMR}$ $\left(500 \mathrm{MHz}, \mathrm{CDCl}_{3}\right) \delta 4.70-4.67(\mathrm{~m}, 1 \mathrm{H}), 4.58-$ $4.55(\mathrm{~m}, 1 \mathrm{H}), 4.47(\mathrm{dt}, 1 \mathrm{H}, J 16.8,8.4 \mathrm{~Hz}), 2.36$ (td, 1H, J 11.0, $5.6 \mathrm{~Hz}), 2.28$ (t, 2H, J 7.6 Hz), 2.05 $-1.98(\mathrm{~m}, 1 \mathrm{H}), 1.96-1.86(\mathrm{~m}, 1 \mathrm{H}), 1.67-1.59$ $(\mathrm{m}, 8 \mathrm{H}), 1.52-1.45(\mathrm{~m}, 2 \mathrm{H}), 1.43-1.25(\mathrm{~m}, 21 \mathrm{H})$, 1.04 (s, 3H), 0.94 (s, 3H), 0.89 (t, 3H, J 6.1 Hz), $0.86(\mathrm{~s}, 3 \mathrm{H}), 0.84$ (s, 3H), 0.84 (s, 3H), 0.79 (s, 3H); ${ }^{13} \mathrm{C}$ NMR (125 MHz, $\left.\mathrm{CDCl}_{3}\right) \delta 173.59,150.89$, 109.31, 80.63, 55.44, 53.34, 50.39, 48.35, 48.01, $43.00,42.86,40.90,40.01,38.43,38.11,37.85$, $37.13,35.60,34.80,34.27,31.90,31.33,29.88$, $29.67,29.44,29.33,29.24,29.16,27.97,27.47$, $25.17,24.82,23.76,22.66,22.28,20.98,19.29$, $18.23,18.00,16.54,16.15,16.00,14.53,14.05$, 13.85 .

\section{Lupenyl oleate (4b)}

Mass obtained: $13.7 \mathrm{mg}, 61 \%$ yield; rf 0.7 in EtOAc:hexane (20:80); $[\alpha]_{\mathrm{D}}^{25}+8.0\left(c 1.0, \mathrm{CHCl}_{3}\right)$; FTIR (KBr) $v / \mathrm{cm}^{-1} 3068,2926,1730,1452,1174$; ${ }^{1} \mathrm{H}$ NMR (500 MHz, $\left.\mathrm{CDCl}_{3}\right) \delta 5.37-5.33(\mathrm{~m}, 2 \mathrm{H})$, 4.70 (d, 1H, J 2.4 Hz), 4.58 (dd, 1H, J 2.4, 1.4 Hz), 4.48 (dd, 1H, J 11.0, $5.4 \mathrm{~Hz}), 2.43-2.35(\mathrm{~m}, 1 \mathrm{H})$, $2.31-2.27$ (m, 2H), $2.06-1.98(\mathrm{~m}, 3 \mathrm{H}), 1.97-$ $1.87(\mathrm{~m}, 2 \mathrm{H}), 1.70(\mathrm{~s}, 3 \mathrm{H}), 1.68-1.55(\mathrm{~m}, 8 \mathrm{H})$, $1.52-1.45(\mathrm{~m}, 3 \mathrm{H}), 1.44-1.15(\mathrm{~m}, 38 \mathrm{H}), 1.04(\mathrm{~s}$, $3 \mathrm{H}), 0.95(\mathrm{~s}, 3 \mathrm{H}), 0.89$ (t, 3H, J 6.5 Hz), $0.87(\mathrm{~s}$, $3 \mathrm{H}), 0.85(\mathrm{~s}, 3 \mathrm{H}), 0.85(\mathrm{~s}, 3 \mathrm{H}), 0.80(\mathrm{~s}, 3 \mathrm{H}) ;{ }^{13} \mathrm{C}$ NMR (125 MHz, $\left.\mathrm{CDCl}_{3}\right) \delta 173.83,151.12,130.16$, 129.94, 109.53, 80.81, 55.59, 50.54, 48.49, 48.20, $43.19,43.03,41.05,40.19,38.58,38.25,38.03$, $37.29,35.77,35.03,34.42,32.12,32.10,30.03$, $29.96,29.89,29.87,29.71,29.55,29.52,29.51$, $29.35,29.34,29.30,28.16,27.64,27.41,27.35$, $25.34,25.31,23.94,22.87,21.15,19.48,18.40$, $18.19,16.76,16.36,16.17,14.71,14.29$.

\section{Stigmasteryl hexanoate (5a)}

Mass obtained: $29.9 \mathrm{mg}, 90 \%$ yield; rf 0.7 in EtOAc:hexane (20:80); $[\alpha]_{\mathrm{D}}^{25}-37.0\left(c 1.0, \mathrm{CHCl}_{3}\right)$, lit. (Kuksis and Beveridge 1960) $[\alpha]_{\mathrm{D}}^{25}-37.7$ (c 1.0, $\mathrm{CHCl}_{3}$ ); FTIR (KBr) v / $\mathrm{cm}^{-1} 2926,1737,1437$, 
1382; ${ }^{1} \mathrm{H}$ NMR $\left(500 \mathrm{MHz}, \mathrm{CDCl}_{3}\right) \delta 5.38(\mathrm{~d}, 1 \mathrm{H}$, $J 5.0 \mathrm{~Hz}), 5.17$ (dd, 2H, $J$ 15.2, 8.6 Hz), 5.03 (dd, $1 \mathrm{H}, J 15.2,8.7 \mathrm{~Hz}), 4.62$ (tdd, $1 \mathrm{H}, J 11.1,6.8,4.2$ Hz), 2.32 (d, 1H, J $6.9 \mathrm{~Hz}), 2.27$ (t, 2H, J $7.5 \mathrm{~Hz})$, $2.08-1.94(\mathrm{~m}, 4 \mathrm{H}), 1.87-1.83(\mathrm{~m}, 2 \mathrm{H}), 1.75-$ $1.68(\mathrm{~m}, 2 \mathrm{H}), 1.66-1.40(\mathrm{~m}, 12 \mathrm{H}), 1.36-1.25$ $(\mathrm{m}, 6 \mathrm{H}), 1.22$ - $1.12(\mathrm{~m}, 4 \mathrm{H}), 1.04(\mathrm{~s}, 3 \mathrm{H}), 1,04$ (d, $3 \mathrm{H}, J 6.5 \mathrm{~Hz}), 0.91$ (t, 3H, $J 6.7 \mathrm{~Hz}), 0.86$ (d, 3H, $J 6.6 \mathrm{~Hz}), 0.82$ (d, 3H, J 7.4 Hz), 0.80 (d, 3H, J 6.3 $\mathrm{Hz}), 0.71(\mathrm{~s}, 3 \mathrm{H}) ;{ }^{13} \mathrm{C}$ NMR $\left(125 \mathrm{MHz}, \mathrm{CDCl}_{3}\right) \delta$ 212.77, 175.21, 153.53, 149.84, 134.67, 134.44, $134.20,133.71,129.38,129.25,129.10,129.08$, $84.55,84.05,83.00,82.75,82.57,82.49,81.33$, $81.17,77.89,75.80,74.33,73.86,35.43,35.39$, $35.09,31.62,31.61,31.56,31.54,31.50,31.45$, $31.36,31.32,28.42,27.02,26.75,26.65,26.60$, 24.20, 24.19, 24.01, 23.79, 19.84 .

\section{Stigmasteryl oleate (5b)}

Mass obtained: $30.0 \mathrm{mg}, 72 \%$ yield; rf 0.7 in EtOAc:hexane (20:80); $[\alpha]_{\mathrm{D}}^{25}-49.0\left(c 1.0, \mathrm{CHCl}_{3}\right)$, lit. (Kuksis and Beveridge 1960) $[\alpha]_{\mathrm{D}}^{25}-49.9(c$ 1.0, $\mathrm{CHCl}_{3}$ ); FTIR (KBr) $v / \mathrm{cm}^{-1} 3551,1783,1487$, 1183; ${ }^{1} \mathrm{H}$ NMR $\left(500 \mathrm{MHz}, \mathrm{CDCl}_{3}\right) \delta 5.38-5.32$ $(\mathrm{m}, 3 \mathrm{H}), 5.16(\mathrm{dd}, 1 \mathrm{H}, J$ 15.2, $8.7 \mathrm{~Hz}), 5.02$ (dd, $1 \mathrm{H}, J 15.1,8.8 \mathrm{~Hz}), 4.64-4.55(\mathrm{~m}, 1 \mathrm{H}), 2.31$ (d, $2 \mathrm{H}, J 7.1 \mathrm{~Hz}), 2.26$ (t, 2H, J 7.4 Hz), $2.10-1.93$ (m, 6H), $1.89-1.81(\mathrm{~m}, 2 \mathrm{H}), 1.78-1.68(\mathrm{~m}, 1 \mathrm{H})$, $1.64-1.39$ (m, 8H), $1.36-1.22(\mathrm{~m}, 28 \mathrm{H}), 1.20-$ $1.12(\mathrm{~m}, 4 \mathrm{H}), 1.02(\mathrm{~s}, 3 \mathrm{H}), 1,02(\mathrm{~d}, 3 \mathrm{H}, J 6.5 \mathrm{~Hz})$, 0.88 (t, 3H, $J 6.7 \mathrm{~Hz}), 0.85$ (d, 3H, $J 6.6 \mathrm{~Hz}), 0.81$ (d, 3H, J 7.4 Hz), $0.80(\mathrm{~d}, 3 \mathrm{H}, J 6.3 \mathrm{~Hz}), 0.70$ $(\mathrm{s}, 3 \mathrm{H}) ;{ }^{13} \mathrm{C}$ NMR $\left(125 \mathrm{MHz}, \mathrm{CDCl}_{3}\right) \delta 173.26$, 139.71, 138.29, 129.97, 129.75, 129.28, 122.55, $73.67,56.79,55.95,51.23,50.06,42.21,40.48$, $39.63,38.16,37.01,36.61,34.71,31.90,31.89$, $31.88,29.77,29.68,29.52,29.32,29.31,29.15$, $29.09,28.89,27.82,27.21,27.16,25.40,25.05$, $24.35,22.67,21.21,21.07,21.02,19.31,18.98$, $14.10,12.23,12.04$.

\section{$\beta$-Sitosteroyl hexanoate (6a)}

Mass obtained: $30.0 \mathrm{mg}, 86 \%$ yield; rf 0.75 in EtOAc:hexane (20:80); $[\alpha]_{\mathrm{D}}^{25}-36.0\left(c 1.0, \mathrm{CHCl}_{3}\right)$, lit. (Kuksis and Beveridge 1960) $[\alpha]_{\mathrm{D}}^{25}-38.0$ (c 1.0, $\mathrm{CHCl}_{3}$ ); FTIR (KBr) v / $\mathrm{cm}^{-1} 3300,2824,2854$, $1724,1616,1512,1508,1450,1381,1172 ;{ }^{1} \mathrm{H}$ NMR $\left(500 \mathrm{MHz}, \mathrm{CDCl}_{3}\right) \delta 5.30(\mathrm{~s}, 1 \mathrm{H}), 4.70-$ $4.58(\mathrm{~m}, 1 \mathrm{H}), 2.32(\mathrm{~d}, 2 \mathrm{H}, J 7.0 \mathrm{~Hz}), 2.27(\mathrm{t}, 2 \mathrm{H}, J$ $7.6 \mathrm{~Hz}), 2.08-1.94(\mathrm{~m}, 4 \mathrm{H}), 1.90-1.80(\mathrm{~m}, 4 \mathrm{H})$, $1.70-1.45(\mathrm{~m}, 10 \mathrm{H}), 1.40-1.25(\mathrm{~m}, 9 \mathrm{H}), 1.25$ - $1.08(\mathrm{~m}, 4 \mathrm{H}), 1.04-1.02(\mathrm{~m}, 6 \mathrm{H}), 0.94-0.88$ $(\mathrm{m}, 6 \mathrm{H}), 0.88-0.78(\mathrm{~m}, 6 \mathrm{H}), 0.68(\mathrm{~s}, 3 \mathrm{H}) ;{ }^{13} \mathrm{C}$ NMR $\left(125 \mathrm{MHz}, \mathrm{CDCl}_{3}\right) \delta 173.48,139.92,138.49$, 129.48, 122.75, 77.45, 77.19, 76.94, 73.86, 56.99, $56.90,56.30,56.25,56.15,51.43,50.26,50.24$, $46.05,42.51,42.41,40.68,39.93,39.84,39.05$, $38.36,37.21,36.81,36.80,36.35,36.09,34.88$, $34.15,33.91,32.62,32.11,32.07,31.50,30.49$, $29.37,29.10,28.44,28.02,26.31,25.60,24.94$, $24.55,24.49,23.28,22.51,21.41,21.27,21.23$, $20.40,20.00,19.51,19.24,19.18,18.97,18.90$, $18.45,15.57,14.11,12.43,12.24,12.18,12.05$.

\section{$\beta$-Sitosteroyl oleate (6b)}

Mass obtained: $40.0 \mathrm{mg}, 87 \%$ yield; rf 0.65 in EtOAc:hexane (20:80); $[\alpha]_{\mathrm{D}}^{25}-28.0\left(c 1.0, \mathrm{CHCl}_{3}\right)$, lit. (Kuksis and Beveridge 1960) $[\alpha]_{\mathrm{D}}^{25}-28.0$ (c 1.0, $\mathrm{CHCl}_{3}$ ); FTIR (KBr) $v / \mathrm{cm}^{-1} 2927,1735,1485$, 1248; ${ }^{1} \mathrm{H}$ NMR (500 MHz, $\mathrm{CDCl}_{3}$ ) $\delta 5.42-5.29$ $(\mathrm{m}, 3 \mathrm{H}), 4.63-4.55(\mathrm{~m}, 1 \mathrm{H}), 2.31(\mathrm{~d}, 2 \mathrm{H}, J 7.1 \mathrm{~Hz})$, $2.26(\mathrm{t}, 2 \mathrm{H}, J 7.5 \mathrm{~Hz}), 2.10-1.92(\mathrm{~m}, 8 \mathrm{H}), 1.90$ - $1.80(\mathrm{~m}, 4 \mathrm{H}), 1.66-1.42(\mathrm{~m}, 11 \mathrm{H}), 1.38-1.24$ $(\mathrm{m}, 26 \mathrm{H}), 1.20-1.10(\mathrm{~m}, 4 \mathrm{H}), 1.04-1.01(\mathrm{~m}, 6 \mathrm{H})$, $0.94-0.78(\mathrm{~m}, 12 \mathrm{H}), 0.68(\mathrm{~s}, 3 \mathrm{H}) ;{ }^{13} \mathrm{C}$ NMR $(125$ $\left.\mathrm{MHz}, \mathrm{CDCl}_{3}\right) \delta 173.11,139.68,138.29,129.94$, 129.71, 129.29, 122.54, 77.27, 77.02, 76.76, 73.63, 56.80, 56.71, 56.13, 56.08, 56.02, 55.97, 51.25, $50.09,50.07,45.86,42.32,42.21,40.49,39.76$, $39.66,39.10,38.86,38.18,37.03,36.61,36.59$, $36.21,36.16,35.90,34.68,33.96,33.72,32.41$, $31.92,31.89,31.48,30.62,30.33,29.78,29.69$, 
29.54, 29.47, 29.33, 29.20, 29.17, 29.10, 28.90, $28.52,28.25,28.19,27.83,27.22,27.17,26.15$.

\section{TUMOR CELL CULTURE AND CYTOTOXIC} ACTIVITY

The tested tumor cell lines (leukemia HL-60, colon adenocarcinoma HCT-116, ovarian carcinoma OVCAR-8, prostate carcinoma PC-3 and glioblastoma SF-295) were kindly donated by the National Cancer Institute (Bethesda, MD, USA). Cells were maintained in RPMI 1640 medium supplemented with $10 \%$ fetal bovine serum, $2 \mathrm{mM}$ glutamine, $100 \mathrm{U} \mathrm{mL}^{-1}$ penicillin, and $100 \mu \mathrm{g} \mathrm{mL}^{-1}$ streptomycin at $37^{\circ} \mathrm{C}$ with $5 \% \mathrm{CO}_{2}$. The cytotoxicity of the compounds was initially tested the against three solid human tumor cell lines: HCT-116 (colon adenocarcinoma), SF-295 (glioblastoma) and OVCAR-8 (ovarian carcinoma) to evaluate the cell growth inhibition using the 3-(4,5- dimethyl2-thiazolyl-2,5-diphenyl-2H-tetrazolium bromide) (MTT) reduction assay (Mossman 1983). Cells were plated in 96 -well plates $\left(0.1 \times 106\right.$ cell $\mathrm{mL}^{-1}$ for OVCAR -8 and SF-295 lines and at $0.7 \times 105$ cell $\mathrm{mL}^{-1}$ for the HCT-116 line in $100 \mu \mathrm{L}$ of medium) and compounds $\left(25 \mu \mathrm{g} \mathrm{mL}^{-1}\right)$ were dissolved in DMSO, added to each well using the HTS - highthroughput screening-biomek 3000-Beckman Coulter (Inc. Fullerton, California, USA). After $69 \mathrm{~h}$ of incubation, the supernatant was replaced by fresh medium containing MTT $\left(0.5 \mathrm{mg} \mathrm{mL}^{-1}\right)$. Three hours later, the MTT formazan product was dissolved in $150 \mu \mathrm{L}$ of DMSO and the absorbance was measured at $595 \mathrm{~nm}$ (DTX 880 Multimode Detector, Beckman Coulter, Inc. Fullerton, CA, USA).

The $\mathrm{IC}_{50}$ value of active samples were determined by MTT assay using increasing

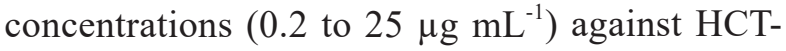
116, SF-295, HL-60 (leukemia) and PC-3 (prostate carcinoma) cells lines after 72 hours of incubation. Doxorubicin was used as the positive control. Control groups received the same amount of
DMSO. The IC50 value and their 95\% confidence intervals (CI 95\%) were obtained by non-linear regression using the GraphPad Software 5.0.

\section{RESULTS AND DISCUSSION}

Our work began by choose of some representative natural products as lead compounds (2-6, Fig. 2). These findings led to betulinic acid ( $3 \beta$-hydroxylup-20-(29)-en-28-oic acid) (2) as one of natural compounds employed. The therapeutical use of this active natural compound as anticancer agent is a highly-investigated research field (Gheorgheosu et al. 2014, Jonnalagadda et al. 2013), and became itself a natural candidate to further chemical modifications. Although the commercial betulinic acid can be easily prepared from common natural sources, its complete synthesis is not available. Other pentacyclic triterpenic acid that has recently attracted a rising attention for its multifunctional anticancer activity is ursolic acid ( $3 \beta$-hydroxy12-urs-12-ene-28-oic acid) (3) (Chen et al. 2015, Wosniak et al. 2015). Inhibition (Kuttan et al. 2011) or cancer prevention (Shanmugam et al. 2013) are some of its properties. Lupeol (3ß)-lup-20-(29)-en3-ol (4) is another triterpene which has gained wide attraction as a therapeutic and chemopreventive agent for treatment of cancer (Saleem 2009), which was also used as template for esters transformations and isolated as an ester derivative as well (Brum et al. 1998). Besides these triperpenic structures, some phytosterols were also used as lead compounds in our study. It class of compounds have been proposed to offer protection against cardiovascular diseases and cancer (Bradford and Awad 2007). Among most common phytosterols, stigmasterol $(3 \beta, 20 R, 22 E, 24 S)$-stigmasta-5,22-dien-3-ol (5) and $\beta$-sitosterol $(3 \beta, 20 R, 24 R)$-stigmast-5-en-3-ol (6) were employed. While the former was already tested against cancer in natural form (Ali et al. 2015, Ghosh et al. 2011), the latter showed effect on tumor cells in ester form (Rathee et al. 2012). 


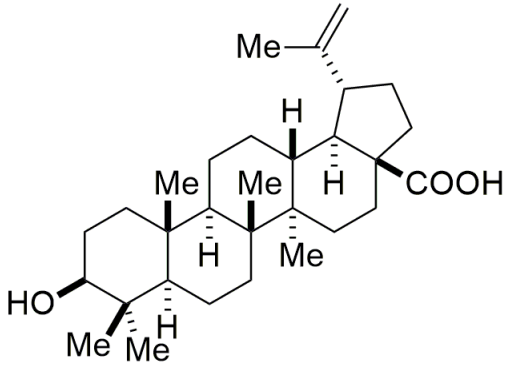

2

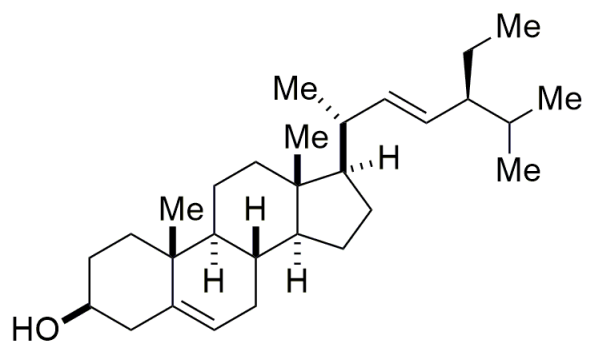

5

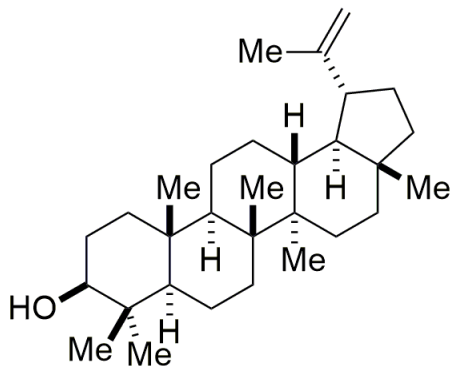

4

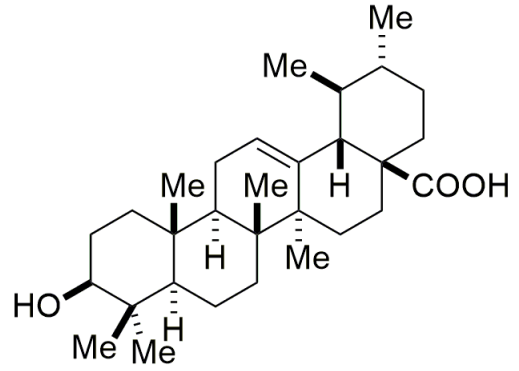

3

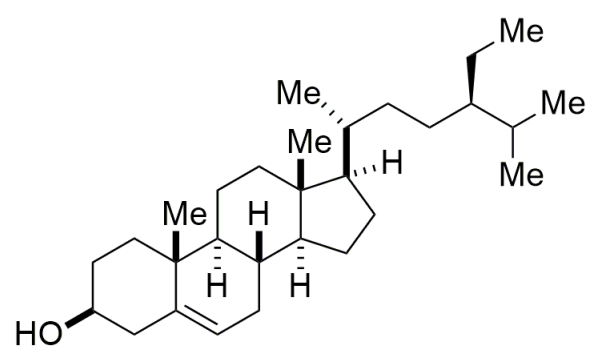

6

Figure 2 - Structures of betulinic and ursolic acids ( 2 and 3, respectively), lupeol (4), stigmasterol (5) and $\beta$-sitosterol (6).

Despite the esters derivatives of phytosterols (Kuksis and Beveridge 1960), and of oleic ester of betulinic acid (Nakagawa-Goto et al. 2009) and lupeol (Chakraborty and Rangari 2011) had already been synthesized, no evaluation of their biological activity against the cancer cells were performed.

The synthesis of hexanoic and oleic ester derivatives from natural terpenes were accomplished by use of Steglich methodology (Neises and Steglich 1978). Treatment of a dichloromethane solution of natural substrate, catalyst (DMAP: 4-N,N-dimethylaminopyridine) and diisopropyl carbodiimide (DIC) with a solution of acid at room temperature led to respective esters (Scheme 1) in moderate to good yields (61-95\%) after $24-48 \mathrm{~h}$ under removal of solid by filtration, concentration and purification by column chromatography in silica-gel (Pilli et al. 2000).

The synthesis was corroborated as described to esters derivatives of betulinic acid 2 . The IR spectra of the derivative showed absorption band at $1728 \mathrm{~cm}^{-1}$, characteristic to $\mathrm{C}=\mathrm{O}$ of ester, and maintenance of acid absorption band between $3600-2600 \mathrm{~cm}^{-1}$ were indicative of esterification. In the ${ }^{1} \mathrm{H}$ NMR spectra the H-3 upshielded signal (from $\delta_{\mathrm{H}} 3.45$ in 2 (Chichewicz and Kouzi 2004) to $\delta_{\mathrm{H}} 4.48$ in 2a) was indicative of the esterification at C-3, and upshielding effect on C-3 in ${ }^{13} \mathrm{C}$ NMR (from $\delta_{\mathrm{C}} 78.1$ in $\mathbf{2}$ to $\delta_{\mathrm{C}} 80.3$ in $\mathbf{2 a}$ ) as well. The presence of side chain was corroborated by appearance of characteristic signal in ${ }^{1} \mathrm{H}$ NMR spectra (triplet at $\delta_{\mathrm{H}} 0.90$ due terminal $\mathrm{CH}_{3}$ ) and ${ }^{13} \mathrm{C}$ NMR as well (signal at $\delta_{\mathrm{C}} 173.5$ due carbonyl ester, besides total of 36 signals for 2a). The IR spectra of oleic derivative $\mathbf{2 b}$ showed a new band at 1732 $\mathrm{cm}^{-1}$ indicating esterification at C-3. Upshielding of H-3 signal from $\delta_{\mathrm{H}} 3.45$ in $\mathbf{2}$ to $\delta_{\mathrm{H}} 4.48$ in $\mathbf{2 b}$ in the ${ }^{1} \mathrm{H}$ NMR corroborated with the presence of an ester bearing C-3. Same effect was observed to $\mathrm{C}-3$ in ${ }^{13} \mathrm{C}$ NMR: upshielding $\mathrm{C} 3$ signal from $\delta_{\mathrm{C}}$ 


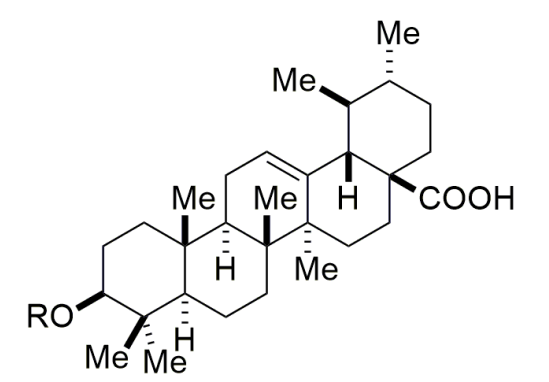

— R=H (ursolic acid 3)

$\rightarrow \mathrm{R}=$ hexanoate $(\mathbf{3 a})(90 \%)$

$\mathrm{R}=$ oleate $(3 \mathbf{b})(90 \%)$

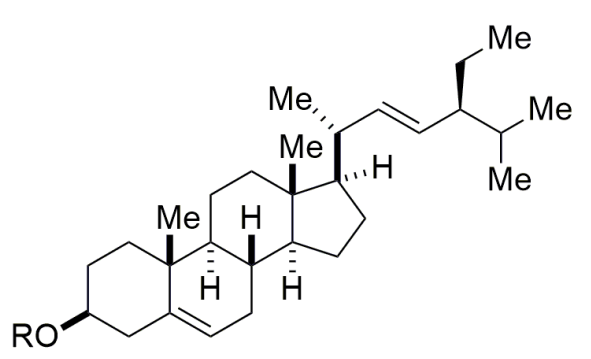

$\begin{aligned} \mathrm{R} & =\mathrm{H} \text { (stigmasterol } \mathbf{5}) \\ \longrightarrow \mathrm{R} & =\text { hexanoate }(\mathbf{5 a})(90 \%) \\ \mathrm{R} & =\text { oleate }(\mathbf{5 b})(72 \%)\end{aligned}$

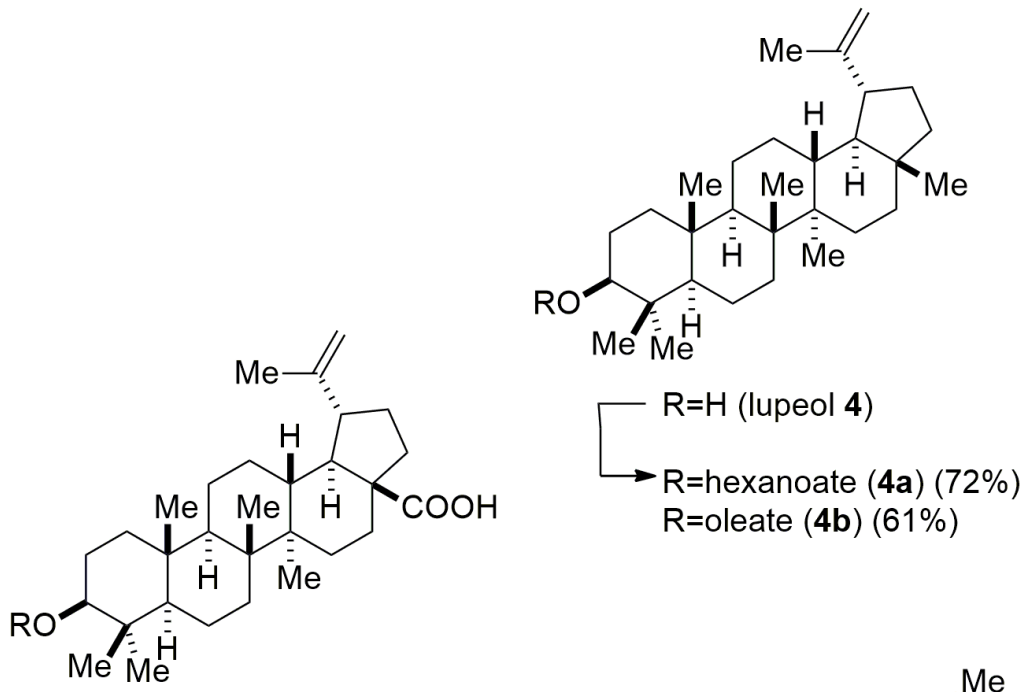

R=H (betulinic acid 2)

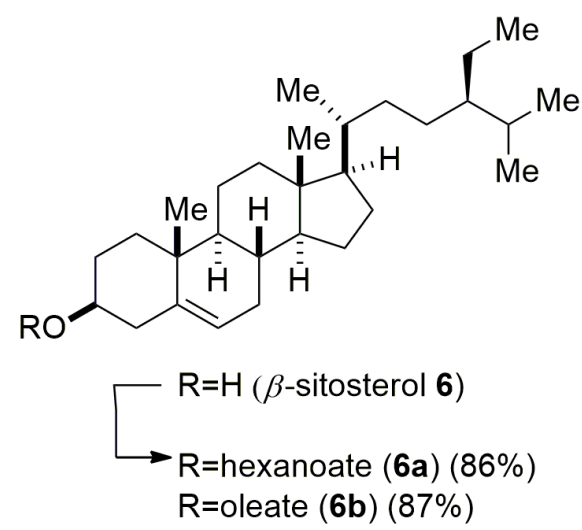

Scheme 1 - Condition to syntheses of esters: DMAP (2 eq.), DIC (2 eq.) in $\mathrm{CH}_{2} \mathrm{Cl}_{2}$, then acid (2 eq.) in $\mathrm{CH}_{2} \mathrm{Cl}_{2}, 24-48 \mathrm{~h}$.

78.1 in $\mathbf{2}$ to $\delta_{\mathrm{C}} 80.6$ in $\mathbf{2 b}$. Side chain was assured by characteristics oleic signals in ${ }^{1} \mathrm{H}$ NMR spectra (olefinic signals at $\delta_{\mathrm{H}}$ 5.38-5.31, vicinal carbonyl $\mathrm{CH}_{2} \delta_{\mathrm{H}} 2.33-2.26$, allylic protons at $\delta_{\mathrm{H}} 2.08-1.91$ and others signals of the aliphatic chain). In ${ }^{13} \mathrm{C}$ NMR spectra new olefinic signals at $\delta_{\mathrm{C}} 130.0$ and 129.7 and all others chains signals at $\delta_{C} 20-40$ region and $\delta_{\mathrm{C}} 14.1$ and 14.7 for terminal methyl showed esterification was accomplished in $\mathbf{2} \mathbf{b}$. The compounds 3a,b-6a,b had their syntheses corroborated as similar as described to $\mathbf{2 a , b}$ (see Figures S1-S40-Supplementary Material).

The ester derivatives $\mathbf{2} \mathbf{a}, \mathbf{b}-\mathbf{6} \mathbf{6} \mathbf{a}, \mathbf{b}$ have their cytotoxic effect evaluated using the MTT assay after $72 \mathrm{~h}$ incubation (Mossman 1983). Initially, the esters derivatives were tested at concentration of $25 \mu \mathrm{g} \mathrm{mL}^{-1}$ against three solid human tumor cell lines: HCT-116 (colon adenocarcinoma), SF-295 (glioblastoma) and OVCAR-8 (ovarian carcinoma), and only the hexanoate derivative of betulinic acid 2a was found to be active, inhibiting tumor cell growth over than $70 \%$ (see Table SI Supplementary Material). Additionally, compound 2a and its parent triterpene betulinic acid $\mathbf{2}$ were tested at increasing concentrations $(0.2$ to $25 \mu \mathrm{g}$ $\mathrm{mL}^{-1}$ ) for $\mathrm{IC}_{50}$ calculation using HCT-116, SF-295, HL-60 (leukemia) and PC-3 (prostate carcinoma) cells (Table I. Results for all compounds are shown in Table SII). Betulinic acid $\mathbf{6}$ was more active than its hexanoate derivative $\mathbf{2 a}$, exhibiting $\mathrm{IC}_{50}$ values ranging from $0.84 \mu \mathrm{g} \mathrm{mL}^{-1}(1.84 \mu \mathrm{M})$ in HL-60 cells to $6.10 \mu \mathrm{g} \mathrm{mL}^{-1}(13.36 \mu \mathrm{M})$ in SF-295 cells. 
TABLE I

Cytotoxic activity of betulinic acid 2 and its hexanoate derivative 2a against HCT-116 (colon adenocarcinoma), SF295 (glioblastoma), HL-60 (leukemia) and PC-3 (prostate carcinoma) cells using MTT assay after $72 \mathrm{~h}$ incubation. Doxorubicin was used as positive control.

\begin{tabular}{ccccc}
\hline \multirow{2}{*}{ Compound } & HL-60 & HCT-116 & SF-295 & PC-3 \\
\cline { 2 - 5 } & \multicolumn{4}{c}{$\mathrm{IC}_{50}(95 \%$ confidence interval $) \mu \mathrm{g} \mathrm{mL} \mathrm{mL}^{-1}(\mathrm{mM})$} \\
\hline \multirow{2}{*}{$\mathbf{2}$} & $0.84(1.84)$ & $2.05(4.49)$ & $6.10(13.36)$ & $1.78(3.9)$ \\
& $(0.74-0.97)$ & $(1.73-2.42)$ & $(4.89-7.62)$ & $(1.42-2.23)$ \\
\multirow{2}{*}{ Doxorubicin } & $10.86(19.57)$ & $9.50(17.12)$ & $17.43(31.41)$ & $14.17(25.54)$ \\
& $(8.54-13.81)$ & $(8.31-10.88)$ & $(12.91-23.54)$ & $(10.31-19.47)$ \\
& $0.02(0.03)$ & $0.12(0.21)$ & $0.24(0.41)$ & $0.26(0.44)$ \\
\hline
\end{tabular}

$\mathrm{IC}_{50}$ values and its $95 \%$ confidence interval were obtained by non-linear regression from two independent experiments performed in duplicate.

The hexanoate derivative 2a exhibited $\mathrm{IC}_{50}$ values ranging from $9.50 \mu \mathrm{g} \mathrm{mL}^{-1}(17.12 \mu \mathrm{M})$ in HCT116 cells to $17.43 \mu \mathrm{g} \mathrm{mL}^{-1}(31.41 \mu \mathrm{M})$ in $\mathrm{SF}-295$ cells (Table I). The in vitro antitumor cytotoxicity of betulinic acid has been extensively studied against several human cancer cell lines including neuroblastoma, glioblastoma, melanoma, leukemia as well as several carcinomas, like head and neck, colon, breast, lung, prostate, renal cell, ovarian and cervix carcinoma, with $\mathrm{IC}_{50}$ values range 1.1 to 16 $\mu \mathrm{g} \mathrm{mL} \mathrm{mL}^{-1}$ depending of tested cell line (Zuco et al. 2002, Hata et al. 2003, Fulda et al. 1997, 1999, Thurnher et al. 2003, Ehrhardt et al. 2004, Manoj et al. 2010). The cytotoxic potential of betulinic acid 2 isolated from Mimosa caesalpiniifolia showed inhibition of cell proliferation over than $86.5 \%$ against HCT-116, SF-295 and OVCAR-8 (Monção et al.2015). Therefore, our results confirm previous findings in the literature.

In conclusion, this article described the synthesis of a series of novel hexanoate and oleate esters from some natural products (stigmasterol, $\beta$-sitosterol, lupeol, and betulinic and ursolic acids), and a cytotoxic panel cells against human ovarian carcinoma OVCAR-8, human gliobastoma SF-295, and human colon carcinoma HCT-116 tumor cell line using an in vitro cytotoxicity assay was performed. Among these compounds, only hexanoic betulinic acid derivative displayed moderate cytotoxic activity and it was efficacious against all tumor cell lines employed (SF-295, HCT-116, PC-3 and HL-60), but it was indeed consistently less active than betulinic acid itself. These preliminary results showed that the synthesis of new derivatives is required to improve anticancer in vitro anticancer activity of the tested compounds.

\section{ACKNOWLEDGMENTS}

The authors thanks Fundação de Amparo à Pesquisa do Estado da Bahia (FAPESB) and Conselho Nacional de Desenvolvimento Científico e Tecnológico (CNPq) for the financial support, the National Cancer Institute (Bethesda, MD, USA) for donating the tumor cell lines used in this study and Laboratório Baiano de Ressonância Magnética Nuclear (LABAREMN, UFBA) for recorded the NMR spectra.

\section{REFERENCES}

ALI H, DIXIT S, ALI D, ALQAHTANI SM, ALKAHTANI S AND ALARIFI S. 2015. Isolation and evaluation of anticancer efficacy of stigmasterol in a mouse model of DMBA-induced skin carcinoma. Drug Des Dev Ther 9: 2793-2800.

AZIMOVA SS. 2013. Occurrence of sesquiterpene esters in plant species, in: Azimova AS and Saidkhodzhaev AI (Eds), Natural Compounds: Natural Sesquiterpenes Esters Plant Sources, Structure and Properties. Springer, New York, p. i-lxxxv. 
BRADFORD PG AND AWAD AB. 2007. Phytosterols as anticancer compounds. Mol Nutr Food Res 51: 161-170.

BRUM RL, HONDA NK, HESS SC, CAVALHEIRO AJ AND MONACHE FD. 1998. Acyl lupeols from Cnidoscolus vitifolius. Phytochemistry 49: 1127-1128.

CARVALHO MG, VELLOSO CRX, BRAZ-FILHO R AND DA COSTA WF. 2001. Acyl-lupeol esters from Parahancornia amapa (Apocynaceae). J Braz Chem Soc 12: 556-559.

CHAKRABORTY AK AND RANGARI VD. 2011. Semisynthetic modification and immunomodulatory activity studies of $19 \alpha-\mathrm{H}$ lupeol esters. Pharm Sinica 2: 198-211.

CHEN H, GAO Y, WANG A, ZHOU X, ZHENG Y AND ZHOU J. 2015. Evolution in medicinal chemistry of ursolic acid derivatives as anticancer agents. Eur J Med Chem 92: 648-655.

CHICHEWICZ RH AND KOUZI SA. 2004. Chemistry, biological activity, and chemotherapeutic potential of betulinic acid for the prevention and treatment of cancer and HIV infection. Med Res Rev 42: 90-114.

DAVID JP, DA SILVA EF, DE MOURA DL, GUEDES MLS, ASSUNÇÃO RJ AND DAVID JM. 2001. Lignans and triterpenes from cytotoxic extract of Eriope blanchetii. Quim Nova 24: 730-733.

EHRHARDT H, FULDA S, FUHRER M, DEBATIN KM AND JEREMIAS I. 2004. Betulinic acid-induced apoptosis in leukemia cells. Leukemia 18: 1406-1412.

FULDA S, FRIESEN C, LOS M, SCAFFIDI C, MIER W, BENEDICT M, NUNEZ G, KRAMMER PH, PETER ME AND DEBATIN KM. 1997. Betulinic acid triggers CD95 (APO-1/Fas)- and p53-independent apoptosis via activation of caspases in neuroectodermal tumors. Cancer Res 57: 4956-4964.

FULDA S, JEREMIAS I, STEINER HH, PIETSCH T AND DEBATIN KM. 1999. Betulinic acid: a new cytotoxic agent against malignant brain-tumor cells. Int $\mathrm{J}$ Cancer 82: 435-441.

GHEORGHEOSU D, DUICI O, DEHELEAN C, SOICA C AND MUNTEAN D. 2014. Betulinic acid as a potent and complex antitumor phytochemical: a minireview. Anticancer Agents Med Chem 14: 936-945.

GHOSH T, MAITY TK AND SINGH J. 2011. Evaluation of antitumor activity of stigmasterol, a constituent isolated from Bacopa monnieri Linn aerial parts against Ehrlich Ascites Carcinoma in mice. Orient Pharm Exp Med 11: 41-49.

HATA K, HORI K, OGASAWARA H AND TAKAHASHI S. 2003. Anti-leukemia activities of Lup-28-al-20(29)-en-3one, a lupane triterpene. Toxicol Lett 143: 1-7.

JONNALAGADDA SC, CORSELLO MA AND SLEER CE. 2013. Betulin-betulinic acid natural product based analogs as anti-cancer agents. Anticancer Agents Med Chem 13: 1477-1499.

KUKSIS A AND BEVERIDGE JMR. 1960. Preparation and certain physical properties of some plant steryl esters. J Org Chem 25: 1209-1219.

KUTTAN G, PRATHEESHKUMAR P, MANU KA AND KUTTAN R. 2011. Inhibition of tumor progression by naturally occurring terpenoids. Pharm Biol 49: 995-1007.

MAHATO SB AND KUNDU AP. 1994. ${ }^{13} \mathrm{C}$ NMR Spectra of pentacyclic triterpenoids - a compilation and some salient features. Phytochemistry 37: 1517-1575.

MANOJ K, PANDEY MK, SUNG B AND AGGARWAL BB. 2010. Betulinic acid suppresses STAT3 activation pathway through induction of protein tyrosine phosphatase SHP-1 in human multiple myeloma cells. Int J Cancer 127: 282292.

MONÇÃO NBN, ARAÚJO BQ, SILVA JN, LIMA DJB, FERREIRA PMP, AIROLDI FPS, PESSOA C AND CITÓ AMGL. 2015. Assessing chemical constituents of Mimosa caesalpiniifolia stem bark: possible bioactive components accountable for the cytotoxic effect of $M$. caesalpiniifolia on human tumour cell lines. Molecules 20: 4216-4224.

MOSSMAN T. 1983. Rapid colorimetric assay for cellular growth and survival: application to proliferation and cytotoxicity assays. J Immunol Methods 65: 55-63.

NAKAGAWA-GOTO K, YAMADA K, TANIGUCHI M, TOKUDA H AND LEE KH 2009. Cancer preventive agents 9. Betulinic acid derivatives as potent cancer chemopreventive agents. Bioorg Med Chem Lett 19: 33783381.

NEISES B AND STEGLICH W. 1978. Simple Method for the Esterification of Carboxylic Acids. Angew Chem Int Ed Engl 17: 522-524.

PILLI RA, VICTOR MM AND DE MEIJERE A. 2000. First total synthesis of aspinolide $\mathrm{B}$, a new pentaketide produced by Aspergillus ochraceus. J Org Chem 65: 5910-5916.

QIAN K, NITZ TJ, YU D, ALLAWAY GP, MORRISNATSCHE SL AND LEE KH. 2010. From natural product to clinical trials: Bevirimat, a plant-derived antiAIDS drug, in: Buss AD and Butler MS (Eds), Natural Products Chemistry for Drug Discovery. Royal Society of Chemistry, Cambridge, p. 374-391.

RATHEE P, RATHEE D, RATHEE D AND RATHEE S. 2012. In-vitro cytotoxic activity of beta-sitosterol triacontenate isolated from Capparis decidua (Forsk.) Edgew. Asian Pac J Trop Med 5: 225-230.

SALEEM M. 2009. Lupeol, a novel anti-inflammatory and anti-cancer dietary triterpene. Cancer Lett 285: 109-115.

SHANMUGAM MK, DAI X, KUMAR AP, TAB BK, SETHI G AND BISHAYEE A. 2013. Ursolic acid in cancer prevention and treatment: molecular targets, pharmacokinetics and clinical studies. Biochem Pharmacol 85: 1579-1587. 
SILVA MLE, DAVID JP, SILVA LCRC, SANTOS RAF, DAVID JM, LIMA, LS, REIS OS AND FONTANA R. 2012. Bioactive oleanane, lupane and ursane triterpene acid derivatives. Molecules 17: 12197-12205.

THURNHER D, TURHANI D, PELZMANN M, WANNEMACHER B, KNERER B, FORMANEK M, WACHECK V AND SELZER E. 2003. Betulinic acid: a new cytotoxic compound against malignant head and neck cancer cells. Head Neck 25: 732-740.

WHO - WORLD HEALTH ORGANIZATION. 2016. World cancer statistics for 2012. http://www.who.int/cancer/en/ (accessed 31.03.16).

WOZNIAK L, SKAPSKA S AND MARSZALEK K. 2015. Ursolic acid - a pentacyclic terpenoid with a wide spectrum of pharmacological activities. Molecules 20: 20614-20641.
ZUCO V, SUPINO R, RIGHETTI SC, CLERIS L, MARCHESI E, GAMBACORTI-PASSERINI C AND FORMELLI F. 2002. Selective cytotoxicity of betulinic acid on tumor cell lines, but not on normal cells. Cancer Lett 175: 17-25.

\section{SUPPLEMENTARY MATERIAL}

Table SI - Percentual inhibition of cell growth in three human tumor cell lines. Data are presented as media \pm standard deviation.

Table SII - Cytotoxic activity of triterpenic esters derivatives 6a.b-10a.b against HCT-116 (colon adenocarcinoma), SF295 (glioblastoma), HL-60 (leukemia) and PC-3 (prostate carcinoma) cells using MTT assay after $72 \mathrm{~h}$ incubation. Doxorubicin was used as positive control.

Figures S1-S40 - describing characterization data (IR, 1H and 13C NMR spectra) of compounds $\mathbf{2 a , b - 6 a , b . ~}$ 